\title{
Corrigendum to "6-Gingerol Protects Heart by Suppressing Myocardial Ischemia/Reperfusion Induced Inflammation via the PI3K/Akt-Dependent Mechanism in Rats"
}

\author{
Tongtong Xu $\mathbb{D},{ }^{1}$ Guowei Qin, ${ }^{2}$ Wei Jiang, ${ }^{3}$ Ying Zhao, ${ }^{4}$ Yongnan Xu, ${ }^{5}$ and Xiangwei Lv $\mathbb{D}^{1}$ \\ ${ }^{1}$ Department of Integrated Traditional Chinese and Western Medicine, First Affiliated Hospital of Guilin Medical University, \\ Guilin 541001, Guangxi Zhuang Autonomous Region, China \\ ${ }^{2}$ Department of Science and Technology, Guilin Medical University, Guilin 541004, Guangxi Zhuang Autonomous Region, China \\ ${ }^{3}$ Department of Traditional Chinese Medicine, First Affiliated Hospital of Guilin Medical University, Guilin 541001, \\ Guangxi Zhuang Autonomous Region, China \\ ${ }^{4}$ Department of Respiratory Medicine, Traditional Chinese Medicine Hospital, Xuzhou 221009, Jiangsu, China \\ ${ }^{5}$ Department of Infectious Diseases, First Affiliated Hospital of Guilin Medical University, Guilin 541001, \\ Guangxi Zhuang Autonomous Region, China \\ Correspondence should be addressed to Xiangwei Lv; lvxiangwei910@glmc.edu.cn
}

Received 10 April 2019; Accepted 14 April 2019; Published 18 June 2019

Copyright (C) 2019 Tongtong Xu et al. This is an open access article distributed under the Creative Commons Attribution License, which permits unrestricted use, distribution, and reproduction in any medium, provided the original work is properly cited.

In the article titled "6-Gingerol Protects Heart by Suppressing Myocardial Ischemia/Reperfusion Induced Inflammation via the PI3K/Akt-Dependent Mechanism in Rats" [1], there was an error in the HE detection image of $\mathrm{LY}+6-\mathrm{G}+\mathrm{I} / \mathrm{R}$ group in Figure 4(a). Figure 4 is shown below. 


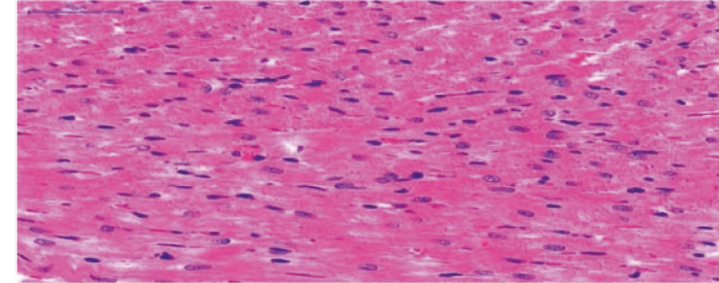

Sham

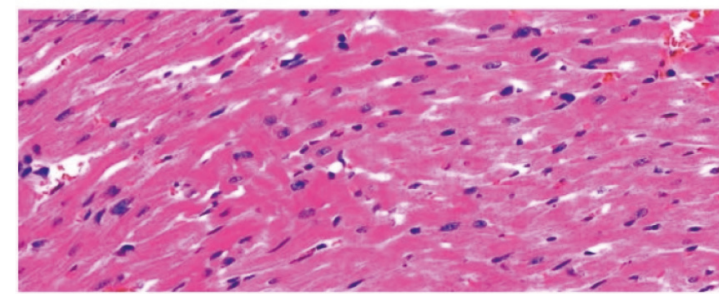

6-G+I/R

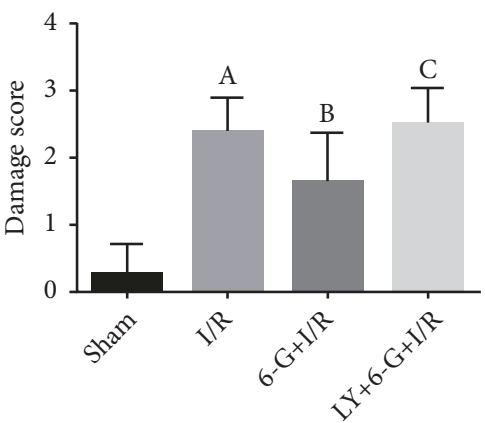

(b)

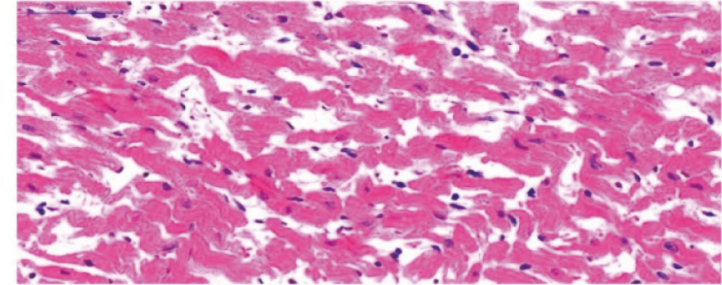

$\mathrm{I} / \mathrm{R}$

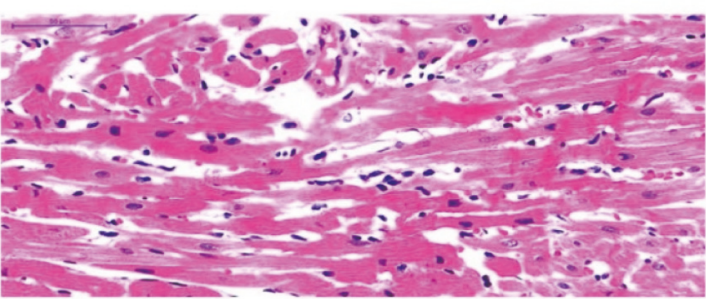

$L Y+6-G+I / R$

(a)

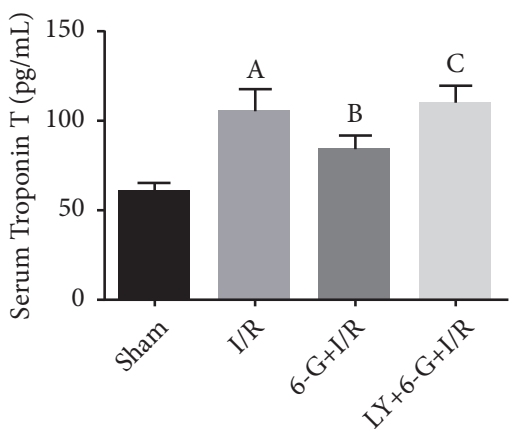

(c)

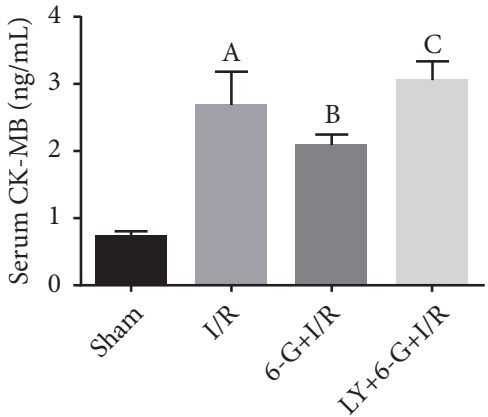

(d)

FIGURE 4: 6-G treatment could alleviate myocardial injury and reduce the level of markers of myocardial injury, but LY294002 could reverse the protecting role of $6-\mathrm{G}$ in myocardium ( $\mathrm{n}=8$ for each group). Note that ${ }^{A} \mathrm{P}<0.05$ against the Sham group; ${ }^{B} \mathrm{P}<0.05$ against the I/R group; and ${ }^{C} P<0.05$ against the $6-\mathrm{G}+\mathrm{I} / \mathrm{R}$ group.

\section{References}

[1] T. Xu, G. Qin, W. Jiang, Y. Zhao, Y. Xu, and X. Lv, "6-gingerol protects heart by suppressing myocardial ischemia/reperfusion induced inflammation via the pi3k/akt-dependent mechanism in rats," Evidence-Based Complementary and Alternative Medicine, vol. 2018, Article ID 6209679, 9 pages, 2018. 


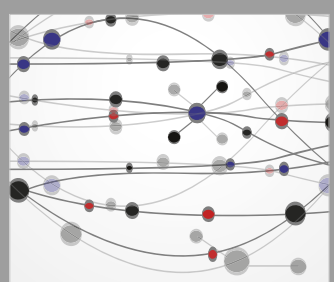

The Scientific World Journal
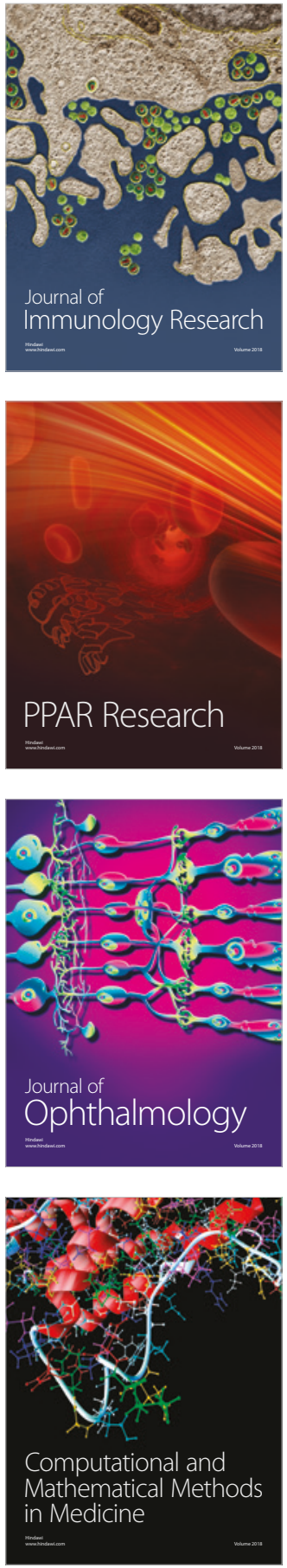

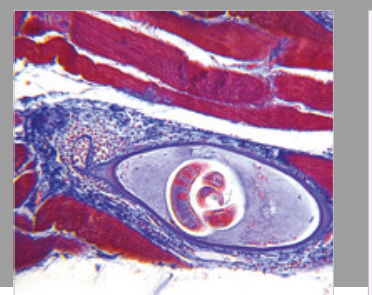

Gastroenterology Research and Practice

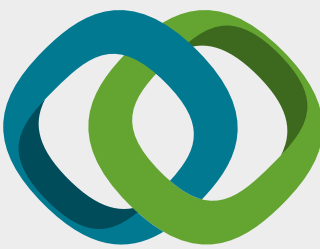

\section{Hindawi}

Submit your manuscripts at

www.hindawi.com
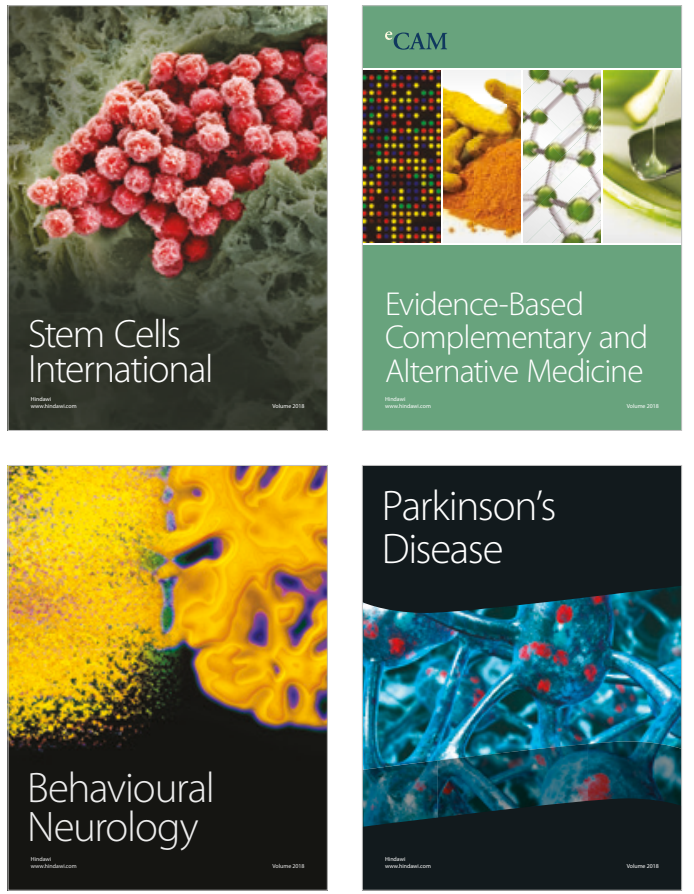

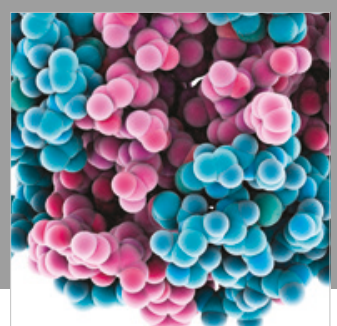

ournal of

Diabetes Research

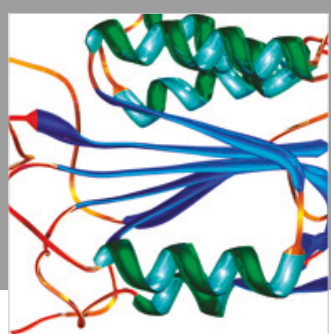

Disease Markers
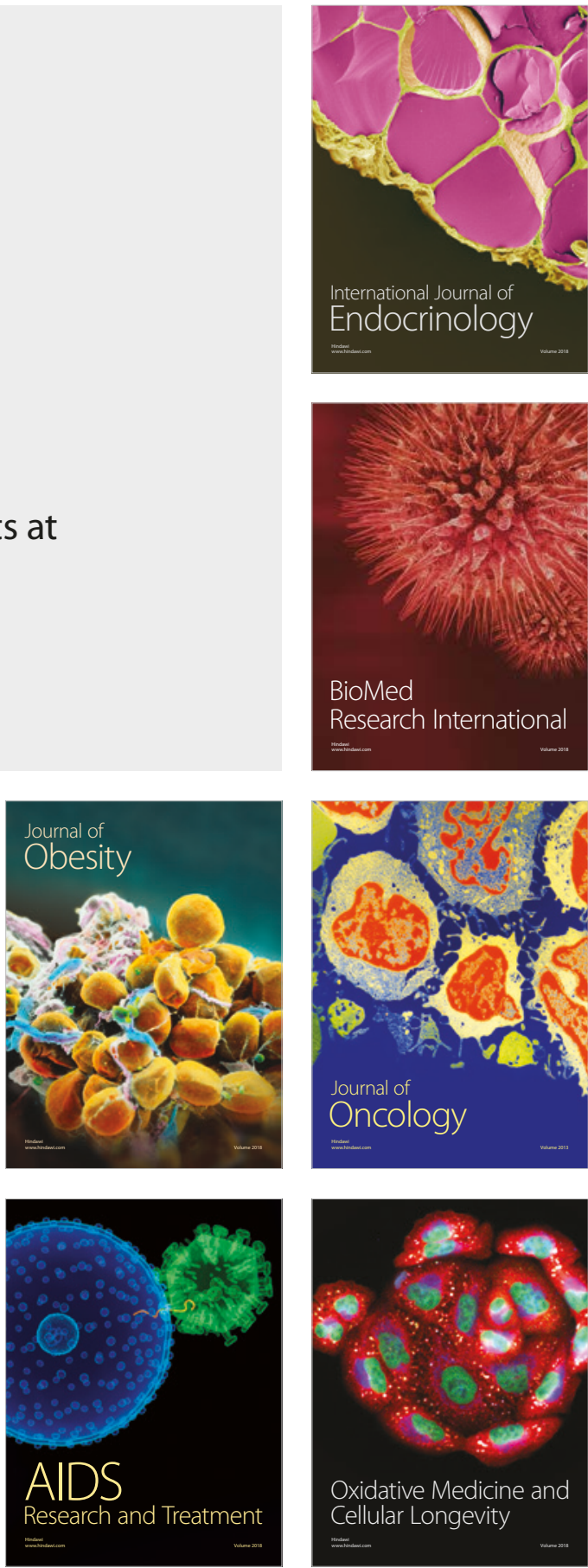